\title{
7 Veränderung von Zugang zu Land im Kontext von land grabbing
}

In der Analyse meiner Fälle geht es mir um eine theoriegeleitete, verstehende Interpretation der erhobenen Daten mit dem Ziel, empirisch begründete Erklärungsansätze zum Zusammenhang des Handels traditioneller Autoritäten und Konflikten um Zugang zu Land zu entwickeln. Der Ausgangspunkt der Analyse ist die Annahme, dass Konflikte im Kontext von land grabbing eng mit Veränderungen der Kontrolle des Zugangs zu Land sowie der Möglichkeiten, Zugang zu Land zu erlangen, verknüpft sind. Zugang zu Land zu haben bedeutet, einen Nutzen aus Land ziehen zu können. Kontrolle über den Zugang $\mathrm{zu}$ Land ausüben zu können bedeutet, bestimmen zu können, ob und welche Akteure einen Nutzen aus Land ziehen können. Entsprechend erlangen Akteure Zugang zu Land, indem sie sich die Kontrolle darüber aneignen oder indem sie Vereinbarungen mit anderen Akteuren treffen, die Zugang zu Land kontrollieren.

Für die Analyse von Konflikten um Zugang ist es zentral, die einzelnen Mechanismen, über die unterschiedliche Akteure Zugang zu Land erlangen und kontrollieren, zu identifizieren. Aufgrund meines Forschungsfokus auf Konflikte im Kontext der großflächigen Vergabe von Land an Investoren gilt es anschließend herauszuarbeiten, inwiefern sich die Möglichkeiten auf die zuvor identifizierten Mechanismen zurückzugreifen in diesem Zusammenhang verändern. Diesen Fragen gehe ich mit Bezug auf die Konflikte um die agrarindustriellen Projekte ScanFarm und BioFuel Africa im Folgenden nach. Zunächst stelle ich dar, wie die Kontrolle von Zugang zu Land vor der Vergabe des Lands an die Unternehmen ausgestaltet war und über welche Mechanismen Landnutzer*innen Zugang zu Land erlangen konnten. Anschließend zeige ich, wie sich Kontrolle über Zugang zu Land sowie die Möglichkeiten Zugang zu Land zu erlangen im Kontext der großflächigen Landvergabe verändert haben. 


\subsection{Kontrolle über Zugang zu Land vor der Landvergabe}

In beiden Fallregionen sind nach traditionellem Recht die paramount chiefs pro forma die Verwalter des Lands. Landnutzer*innen, sub-chiefs und staatliche Akteure bezeichnen sie gemeinhin auch als Eigentümer des Lands (owner of the land) ${ }^{1}$. Das bedeutet jedoch nicht, dass die paramount chiefs vor der Vergabe des Lands an ScanFarm und BioFuel Africa die alleinige Kontrolle über den Zugang zu Land ausübten.

Paramount chiefs übertragen unter Rückbezug auf Gewohnheitsrecht in beiden Fällen die Verwaltung des Lands - und damit auch teilweise die Kontrolle des Zugangs zu Land - an ihnen untergebene sub-chiefs, die aus der Abstammungslinie der Gründer*innen der in der Gegend lebenden Gemeinschaft (royal family) abstammen. In der Regel verwalten sub-chiefs Land in der Gegend, in der sie selbst auch leben ${ }^{2}$. Die Zuständigkeit für die Verwaltung des Lands erhalten sie im Rahmen ihrer Amtseinführung durch den paramount chief. Fortan kontrollieren sub-chiefs in ihrem Verwaltungsbereich den Zugang zu Land für die subsistenzwirtschaftliche Nutzung durch Mitglieder der Gemeinschaft oder Migrant*innen. Das durch sub-chiefs verwaltete Land besteht aus stool land und community land. Stool land sind Flächen, auf die der traditional council, dem alle chiefs einer traditionell verwalteten Gegend (traditional area) angehören, gewohnheitsrechtlichen Anspruch erhebt. Community land bezeichnet Flächen, die zur gemeinschaftlichen oder individuellen Nutzung durch Mitglieder der Gemeinschaft oder Migrant"innen zur Verfügung stehen. Sub-chiefs kontrollieren Zugang zu stool land und community Land, indem sie Nutzungsrechte für subsistenzwirtschaftliche $Z$ wecke vergeben. Diese Nutzungsrechte können sie Landnutzer"innen aus der Gemeinschaft, die

$1 \quad$ Landnutzer*innen aus Jimle und Cumani, Gruppendiskussion am 10.5.2016; Landnutzer und settler-chief aus Parachanayilli, Gruppendiskussion am 13.5.2016; Landnutzer*innen und sub-chief von Tuya, Gruppendiskussion am 14.5.2016; Landnutzerin aus Nsonyameye, Interview am 25.4.2016; Landnutzer aus Dukusen, Interview am 26.4.2016; Mitarbeiterin des Regional Office of the Administrator of Stool Lands der Northern Region, Interview am 4.5.2016; Research Officer des Regional House of Chiefs der Northern Region, Interview am 8.3.2017; Leitender Angestellter BioFuel Africa, Interview am 24.5.2016. am 19.4.2016; Research Officer des Regional House of Chiefs der Northern Region, Interview am 8.3.2017. 
mehr Land benötigen als ihnen bisher zur Verfügung stand oder Migrant*innen zusprechen ${ }^{3}$. Entsprechend haben die sub-chiefs Kenntnisse darüber, welche Flächen in ihrem Verwaltungsbereich wie und durch wen genutzt werden. Den Zugang zu Land für die Subsistenzwirtschaft kontrollieren sub-chiefs somit unabhängig vom paramount chief. Dieser übt hingegen weiterhin Kontrolle über den Zugang zu Land für kommerzielle landwirtschaftliche Vorhaben aus. Dabei kann es sich um großflächige agrarindustrielle Projekte oder um kleinere Vorhaben handeln, die eine deutliche kommerzielle Ausrichtung haben und durch Akteure umgesetzt werden, die nicht in der jeweiligen Gemeinschaft leben. Der paramount chief ist für die endgültige Bewilligung dieser Vorhaben zuständig und erhebt Einnahmen für die Nutzung des Lands, wie Pacht oder seltener Ernteabgaben. Dem Gewohnheitsrecht entsprechend verteilt er einen Teil dieser Einnahmen an die sub-chiefs, in deren Gegend sich das Vorhaben befindet ${ }^{4}$. Der sub-chief wiederum handelt in diesem Kontext als Berater des paramount chiefs und informiert ihn darüber, welches Stück Land für ein entsprechendes Vorhaben zur Verfügung steht ${ }^{5}$. Darüber hinaus ist der paramount chief in der Gegend von ScanFarm auch für die Formalisierung von Landrechten zuständig, die er gemeinsam mit dem sub-chief bewilligt ${ }^{6}$.

Auf Grundlage des Gewohnheitsrechts kontrollieren neben den sub-chiefs in beiden Fallregionen auch teilweise indigene Landnutzer*innen Zugang zu Land. Dies sind Landnutzer*innen, die über permanente traditionelle Landrechte verfügen. Diese Rechte haben sie nicht über zuständige traditionelle Autoritäten, sondern aufgrund ihrer sozialen Zugehörigkeit erlangt. Nach traditionellem Recht bestehen sie ohne zeitliche Begrenzung und können an Dritte temporär vergeben, dauerhaft übertragen oder vererbt werden. Inhaber*innen solcher permanenten traditionellen Landrechte kontrollierten vor der Vergabe des Lands an die Unternehmen ihren eigenen Zugang sowie den Zugang anderer im Rahmen individueller Absprachen wie sharecropping-oder Pachtvereinbarungen.

Sub-chief von Dukusen, Interview am 19.4.2016; Research Officer des Regional House of Chiefs der Northern Region, Interview am 8.3.2017.

4 Registerführer des Agogo Traditional Council, Interview am 14.3.2017.

5 Sub-chief von Nsonyameye, Interview am 20.4.2016; Research Officer des Regional House of Chiefs der Northern Region, Interview am 8.3.2017.

6 Landnutzer aus Nsonyameye und Vorsitzender derAgogoman mma kuo, Interview am 16.3.2017. 
Auch wenn staatliche Akteure, sub-chiefs und Landnutzer*innen die paramount chiefs in beiden Gegenden als Eigentümer des Lands bezeichnen, übten sie vor der Vergabe des Lands an ScanFarm und BioFuel Africa nur begrenzt Kontrolle über den Zugang zu Land aus. Den Zugang für die subsistenzwirtschaftliche Nutzung kontrollierten de facto sub-chiefs sowie Landnutzer*innen mit permanenten traditionellen Landrechten unabhängig von den paramount chiefs. Sie konnten im Kontext der Subsistenzwirtschaft ohne Rücksprache mit den paramount chiefs ihren eigenen Zugang zu Land und denjenigen anderer Akteure bestimmen. Die paramount chiefs kontrollierten Zugang zu Land nur für die kommerzielle Nutzung von Land und - in der Gegend von ScanFarm - im Rahmen der Formalisierung von Landrechten ${ }^{7}$. In beiden Kontexten agierten sie insbesondere als bewilligende Instanz.

\subsection{Zugang zu Land vor der Landvergabe an ScanFarm und BioFuel Africa}

Die Mechanismen, die es Landnutzer*innen in den Gegenden von ScanFarm und BioFuel Africa vor der Vergabe des Lands an die Unternehmen ermöglichten, Zugang zu dem dortigen traditionell verwalteten Land zu erhalten, sind vielfältig. In erster Linie bestimmten rechtebasierte Mechanismen wie traditionelle Rechte oder individuelle Vereinbarungen den Zugang zu Land. Rechtebasierte Mechanismen ermöglichten jedoch den Zugang zu Land nicht allein, sondern im Zusammenspiel mit relationalen und strukturellen Mechanismen. Relevante relationale Mechanismen waren soziale Zugehörigkeit, soziale Beziehungen sowie Netzwerke. Über diese konnte - innerhalb struktureller Grenzen und damit einhergehenden Ungleichheitsverhältnissen - rechtebasierter Zugang zu Land erlangt oder verhandelt werden. Insbesondere die Interpretation und Aushandlung sozialer Zugehörigkeit innerhalb sozialer Beziehungen war in beiden Fällen relevant, um bestehende Landrechtsansprüche zu konsolidieren oder zu erheben. Rechtebasierter Zugang konnte darüber hinaus innerhalb von Beziehungen ausgehandelt werden, indem Rechteinhaber*innen individuelle Vereinbarungen mit Land-

7 Paramount-chief und elders von Tijo, Gruppendiskussion am 8.5.2016; sub-chief von Nsonyameye, Interview am 20.4.2016; Landnutzer aus Nsonyameye und Vorsitzender der Agogoman mma kuo, Interview am 16.3.2017. 
nutzer*innen ohne bestehende Rechte trafen, um letzteren gegen Pacht oder Naturalleistungen Zugang zu Land zu gewähren.

Im Folgenden stelle ich entlang der rechtebasierten, relationalen und strukturellen Zugangsmechanismen die Ausgestaltung des Zugangs zu Land in den Regionen der beiden untersuchten Fälle vor der Landvergabe an die Unternehmen dar. Daran anschließend analysiere ich, inwiefern sich Zugang $\mathrm{zu}$ Land im Kontext der Vergabe des Lands an ScanFarm und BioFuel Africa verändert hat.

\section{Zugang zu Land über rechtebasierte Mechanismen}

Rechtebasierte Mechanismen, die Landnutzer*innen in den Fallregionen vor der Vergabe des Lands an die Unternehmen Zugang zu Land ermöglichten, waren divers und unterschieden sich zwischen den Landnutzer*innen aufgrund strukturell begründeter Ungleichheitsverhältnisse. Rechte variierten insbesondere zwischen indigenen und migrantischen Landnutzer*innen, jedoch auch innerhalb dieser Gruppen.

Indigene Landnutzer*innen erlangen in beiden Fallregionen Zugang zu Land im Wesentlichen über ihre Zugehörigkeit zu der Gruppe, die sich als erste in der Gegend niedergelassen hat (first settlement) und aufgrund dessen traditionelle Landrechtsansprüche erhebt ${ }^{8}$. In der Gegend des ScanFarmProjekts beziehen sich Landnutzer*innen für die Einforderung von Landrechten zudem auf die erstmalige Bestellung des Lands (first cultivation) durch sie selbst oder ihre Vorfahren ${ }^{9}$. Der Rückbezug auf die erste Niederlassung sowie die erste Bestellung des Lands ermöglicht es Landnutzer*innen, Ansprüche auf permanente Zugangsrechte auf Land geltend zu machen. Eine geläufige Bezeichnung für diese permanenten Landrechte ist die des customary free-

$8 \quad$ Landnutzerin aus Nsonyameye, Interview am 25.4.2016; Research Officer des Regional House of Chiefs der Northern Region, Interview am 8.3.2017; paramount-chief und elders von Tijo, Gruppendiskussion am 8.5.2016.

9 Sub-chief von Nsonyameye, Interview am 20.4.2016; Landnutzerin aus Nsonyameye, Interview am 24.4.2016; Landnutzerin aus Nsonyameye, Interview am 24.4.2016; Landnutzerin aus Nsonyameye, Interview am 27.4.2016; Landnutzer aus Nsonyameye, Interview am 28.4.2016; Landnutzerer aus Nsonyameye und Mitglieder der Agogoman mma kuo, Interview am 16.3.2017; Landnutzer aus Nsonyameye und Vorsitzender der Agogoman mma kuo, Interview am 16.3.2017. 
hold, des traditionellen Grundbesitzes ${ }^{10}$. Rechteinhaber*innen, die über einen customary freehold verfügen, können eigenständig über die Nutzung des Lands entscheiden, zahlen an niemanden Abgaben, können es eigenständig verpachten und an ihre Nachkommen vererben. Nach traditionellem Recht bleibt ein customary freehold auch dann bestehen, wenn Rechteinhaber*innen das Land über einen langen Zeitraum brach liegen lassen und auch nicht betreten, um Feuerholz zu sammeln oder darauf befindliche Bäume zu ernten ${ }^{11}$. Diese Rechte bezeichne ich im weiteren Verlauf des Buches als permanente Rechte.

Landnutzer*innen in der Gegend des ScanFarm-Projekts, die sich auf die erste Niederlassung ihrer Vorfahren in der Gegend berufen, um ihre Rechte an Land geltend $\mathrm{zu}$ machen, beziehen sich in der Regel auf einen lange zurückliegenden Zeitpunkt. Sie berichten, ihre Vorfahren seien in eine bis dato unbesiedelte Gegend gekommen, um sich dort niederzulassen und eine Gemeinschaft zu gründen. Da sie die Ersten in der Gegend waren, seien sie Indigene. Traditionelle Autoritäten kommen ebenfalls aus einer der Familien, die sich als erste in einer Gegend niedergelassen haben ${ }^{12}$.

Weitaus häufiger als auf die erste Niederlassung verweisen Landnutzer*innen in der Gegend des ScanFarm-Projekts darauf, dass sie oder ihre unmittelbaren Vorfahren die Ersten gewesen seien, die das Land bestellt haben. Dies seien der Ursprung und die Grundlage ihrer Rechte an Land ${ }^{13}$. Diese Argumentationsweise setzt a priori voraus, dass sie indigene Landnutzer*innen sind. Denn nach traditionellem Recht können sich nur indigene Landnutzer*innen Land aneignen, indem sie es urbar machen und bestellen $^{14}$. In der Gegend von ScanFarm ist es in der Vergangenheit einigen Landnutzer*innen gelungen, über die erstmalige Bestellung des Lands im

10 Landnutzer aus Nsonyameye und Vorsitzender der Agogoman mma kuo, Interview am 16.3.2017.

$11 \quad$ Landnutzer aus Nsonyameye, Interview am 28.4.2016.

12 Landnutzerin aus Nsonyameye, Interview am 25.4.2016.

13 Sub-chief von Nsonyameye, Interview am 20.4.2016; Landnutzerin aus Nsonyameye, Interview am 24.4.2016; Landnutzerin aus Nsonyameye, Interview am 24.4.2016; Landnutzerin aus Nsonyameye, Interview am 27.4.2016; Landnutzer aus Nsonyameye, Interview am 28.4.2016; Landnutzerer aus Nsonyameye und Mitglieder der Agogoman mma kuo, Interview am 16.3.2017; Landnutzer aus Nsonyameye und Vorsitzender der Agogoman mma kuo, Interview am 16.3.2017.

14 Landnutzerin aus Nsonyameye, Interview am 25.4.2016; Landnutzer aus Nsonyameye, Interview am 28.4.2016. 
Laufe der Zeit Zugang zu Landflächen von bis zu 500 acres - etwas mehr als 200 Hektar - zu erlangen ${ }^{15}$. Diese Flächen wurden jedoch nicht dauerhaft bestellt. Um die Böden zu schonen, betreiben die meisten Landnutzer*innen Wanderfeldbau (shifting cultivation), bei dem sie die Äcker regelmäßig brach liegen lassen, damit sich der Boden regenerieren kann.

Im Gegensatz zur ersten Niederlassung bezieht sich der Verweis auf die erste Bestellung des Lands meistens auf eine jüngere Vergangenheit ${ }^{16}$. Landrechtsansprüche, die sich auf eine jüngere Vergangenheit beziehen, werden wenn es zu Konkurrenz um Flächen kommt - eher durch Akteure, die Zugang $\mathrm{zu}$ Land kontrollieren infrage gestellt, als solche mit einer langen Geschichte. Gemeinschaften indigener Landnutzer*innen unter dem Vorsitz eines subchiefs, die erst in der jüngeren Vergangenheit - vor ein bis zwei Generationen - begonnen hatten, Land in einer bestimmten Gegend zu nutzen, wurden immer wieder von in der Hierarchie höher stehenden traditionellen Autoritäten darauf hingewiesen, dass sich ihr Land trotz permanenter Nutzungsrechte unter deren Zuständigkeit befinde ${ }^{17}$. Dies ereignete sich insbesondere im Rahmen der Ernennung eines neuen sub-chiefs durch den paramount chief, der Umsetzung (kleinerer) kommerzieller landwirtschaftlicher Vorhaben oder der Freigabe von traditionell verwaltetem Land als Weideland durch den paramount chief ${ }^{18}$. In keinem der berichteten Fälle hatte dies jedoch im Alltag direkte Auswirkungen für die Landnutzer*innen, deren Zugang zu Land sich dadurch nicht veränderte. Dementsprechend fochten Landnutzer*innen und sub-chiefs solche Aussagen des paramount chiefs nicht an.

In der Gegend des BioFuel Africa-Projekts waren - insbesondere für Frauen - kollektive Rechte an Land ein wichtiger Zugangsmechanismus. Diese Rechte ermöglichten es Frauen, Bäume auf gemeinschaftlich genutzten Flächen zu ernten und die Baumfrüchte für den Eigenbedarf oder den Verkauf weiterzuverarbeiten. Darüber gelang es ihnen ein zusätzliches Einkommen für die Familie zu erwirtschaften ${ }^{19}$.

15 Landnutzer aus Nsonyameye und Vorsitzender der Agogoman mma kuo, Interview am 16.3.2017.

16 Landnutzerin aus Nsonyameye, Interview am 25.4.2016.

17 Landnutzerin aus Nsonyameye, Interview am 25.4.2016.

18 Landnutzerin aus Nsonyameye, Interview am 25.4.2016; Landnutzerer aus Nsonyameye und Mitglieder der Agogoman mma kuo, Interview am 16.3.2017.

19 Landnutzer*innen und settler-chief aus Kpachaa, Gruppendiskussion am 11.5.2016; Landnutzer und settler-chief aus Parachanayilli, Gruppendiskussion am 13.5.2016; Landnutzer*innen und sub-chief von Tuya, Gruppendiskussion am 14.5.2016. 
Ein weiterer rechtebasierter Mechanismus, über den indigene und migrantische Landnutzer*innen in den Fallregionen Zugang zu Land erlangen, sind individuelle Vereinbarungen mit Personen, die über permanente $\mathrm{Zu}$ gangsrechte an Land verfügen. Diese Personen können traditionelle Autoritäten oder indigene Landnutzer*innen sein. Individuelle Vereinbarungen sind - wenn nicht anders abgemacht - zeitlich unbegrenzt. Sie garantieren jedoch keinen dauerhaft verlässlichen Zugang $\mathrm{zu}$ Land, da sie jederzeit nach der Ernte aufgekündigt werden können. Meistens nehmen individuelle Vereinbarungen die Form mündlicher Absprachen an, nur selten halten die beiden Parteien sie schriftlich fest ${ }^{20}$. In beiden Fallregionen sind individuell vereinbarte Zugangsrechte an die Nutzung des Lands geknüpft. Aus der Bestellung des Lands leiten sich wiederum temporäre Rechtsansprüche ab. Diese gelten gleichermaßen für indigene und migrantische Landnutzer*innen. Solange Landnutzer*innen im Rahmen individueller Vereinbarungen Land bestellen, können sie nach traditionellem Recht Ansprüche darauf geltend machen. Im Falle des Verlusts dieses Lands haben sie zum Beispiel ein Anrecht auf die noch ausstehende Ernte oder - beim Verlust der Ernte - auf entsprechende Entschädigungszahlungen ${ }^{21}$. Die Rechte auf Land von Indigenen und Migrant*innen unterscheiden sich jedoch, wenn der Zugang zu Land über eine traditionelle Autorität ermöglicht wurde. Indigenen steht im Fall des Verlusts ihres Lands ein neues Stück Land zu, Migrant*innen haben darauf keinen Anspruch ${ }^{22}$.

Neben Landnutzungsvereinbarungen zwischen traditionellen Autoritäten und Landnutzer*innen ist sharecropping eine gängige Art der individuellen Vereinbarung ${ }^{23}$. Sharecropping-Vereinbarungen ermöglichen es Landnutzer*innen ohne bestehende Landrechtsansprüche, als Pächter*innen Nutzungsrechte zu erlangen. Diese Nutzungsrechte können sie über Inhaber*innen permanenter Landrechte erhalten. Sharecropping verstehe ich als rechtebasierten Zugangsmechanismus, da die beteiligten Akteure aufgrund zuvor bestehender oder neuverhandelter Rechte Zugang zu Land haben. Pächter*innen erlangen Zugang über die Nutzung des Lands, Rechteinha-

\footnotetext{
20 Landnutzer aus Nsonyameye, Interview am 25.4.2016.

21 Landnutzer aus Nsonyameye, Interview am 28.4.2016.

22 Landnutzer aus Nsonyameye, Interview am 25.4.2016.

23 Landnutzerin aus Nsonyameye, Interview am 24.4.2016; Landnutzerin aus Nsonyameye, Interview am 24.4.2016; Landnutzerin aus Nsonyameye, Interview am 27.4.2016.
} 
ber*innen halten ihren Zugang zu Land über die Abgaben der Pächter*innen aufrecht.

Neben sharecropping sind in der Gegend von ScanFarm auch klassische Pachtverträge eine verbreitete Form der individuellen Vereinbarung. Anders als beim sharecropping verlangen Pächter*innen für die Nutzung ihres Lands einen vorher festgesetzten Geldbetrag. Zum Zeitpunkt der Interviews, im April 2016, betrug dieser 200 GHS pro acre und Jahr, was damals umgerechnet knapp $30 €$ entsprach ${ }^{24}$.

$\mathrm{Zu}$ den individuellen Vereinbarungen zähle ich darüber hinaus die Formalisierung traditioneller Landrechtsansprüche. Diese Möglichkeit besteht für indigene Landnutzer*innen mit permanenten Landrechten, die sie schriftlich festhalten und durch traditionelle Autoritäten bestätigen lassen können. Als Formalisierung bezeichne ich die schriftliche Bestätigung mündlich ausgehandelter Rechte. Die Formalisierung traditioneller Landrechte erfolgt durch die schriftliche Bestätigung von Landrechten durch den für die Gegend zuständigen sub-chief sowie den jeweiligen paramount chief ${ }^{25}$. Eine solche Formalisierung ist eine Voraussetzung, wenn Landnutzer*innen traditionelle Landrechte bei der staatlichen Lands Commission registrieren lassen möchten. Sie dient jedoch insbesondere dazu, permanente Investitionen in Land wie bauliche Strukturen zur Weiterverarbeitung von landwirtschaftlichen Erzeugnissen - abzusichern. In der Gegend des BioFuel Africa-Projekts ist die Formalisierung traditioneller Landrechte nicht verbreitet. In derjenigen des ScanFarm-Projekts gibt es sie. Jedoch hat nur einer meiner Interviewpartner von der Formalisierung seiner traditionellen Landrechte Gebrauch gemacht. Mit 500 acres verfügte er über eine verhältnismäßig große Fläche, die er für die kommerzielle Landwirtschaft nutzte und auf der er eine Mühle zur Weiterverarbeitung von Maniok sowie einen Schweinezuchtbertrieb gebaut hat $^{26}$. Subsistenzwirtschaft betreibende Landnutzer*innen mit formalisierten Landrechten sind mir durch meine Forschung nicht bekannt.

24 Landnutzer*innen aus Nsonyameye, Baama \& Dukusen, Gruppendiskussion am 27.4.2016; Landnutzerin aus Nsonyameye, Interview am 25.4.2016; Landnutzer aus Nsonyameye, Interview am 25.4.2016.

25 Landnutzer aus Nsonyameye und Vorsitzender der Agogoman mma kuo, Interview am 16.3.2017.

26 Landnutzer aus Nsonyameye und Vorsitzender der Agogoman mma kuo, Interview am 16.3.2017. 


\section{Zugang zu Land über relationale und strukturelle Mechanismen}

Neben rechtebasierten Zugangsmechanismen sind in beiden Fallstudienregionen relationale und strukturelle Mechanismen für den Zugang zu Land relevant. Relationale und strukturelle Zugangsmechanismen ergeben sich aus den kontextspezifischen Bedingungen, unter denen Landnutzer*innen nach Zugang zu Land streben. Sie bezeichnen die Möglichkeiten, die sich aus diesen Bedingungen in Bezug auf den Zugang zu Land ableiten. Dazu zählen die Aushandlung sozialer und rechtlicher Ordnungen, in dessen Rahmen Akteure nach Zugang zu Land streben sowie Netzwerke und soziale Beziehungen, die Zugang zu Land ermöglichen können. Darüber hinaus beeinflussen Kategorien sozialer Zugehörigkeit wie Alter, Geschlecht, Klasse und Herkunft, die mit strukturell begründeten Ungleichheitsverhältnissen einhergehen, die Möglichkeiten, Rechte an Land einzufordern. Relationale und strukturelle Mechanismen sind eng mit rechtebasierten Zugangsmechanismen verschränkt. Relationale und strukturelle Mechanismen können - zum Beispiel vermittelt über soziale Zugehörigkeit - die Grundlage für die Einforderung von rechtebasiertem Zugang zu Land bilden oder seiner Absicherung dienen, wenn er infrage gestellt wird. Für Akteure, die selbst nicht auf rechtebasierte Zugangsmechanismen zurückgreifen können, erweitern relationale und strukturelle Mechanismen die Möglichkeiten, Zugang zu Land zu erlangen.

In beiden Fallregionen stützen relationale und strukturelle Mechanismen rechtebasierten Zugang zu Land. Landnutzer*innen handeln rechtebasierten Zugang zu Land innerhalb sozialer Beziehungen aus. Die Möglichkeiten der Aushandlung und der Einforderung traditioneller Landrechte ist eng an Kategorien sozialer Zugehörigkeit geknüpft. Zentral für die Aushandlung von rechtebasiertem Zugang zu Land ist in beiden Forschungsregionen die Kategorie Herkunft und damit verbundene Selbst- oder Fremdzuschreibungen der Landnutzer*innen (Indigene/Migrant*innen, Einheimische/Fremde, first comer/late comer). Argumente wie die erste Niederlassung in einer Gegend, die erste Bestellung des Lands oder die Aussage "meine Familie hat schon immer in dieser Gegend gelebt « veranschaulichen dies. Die Wirksamkeit solcher Argumentationsweisen impliziert, dass Indigenität eine gemeinschaftlich anerkannte Voraussetzung für die Einforderung permanenter Landrechte ist. Im Umkehrschluss leitet sich daraus ab, dass Landnutzer*innen aus 
anderen Gegenden nur eingeschränkten Zugang zu Land erhalten können ${ }^{27}$. Die Anerkennung als Indigene* $r$ durch Akteure, die Zugang zu Land kontrollieren, ist somit ein zentraler relationaler Zugangsmechanismus, der Rechte an Land nach sich ziehen kann. Im Umkehrschluss kann über die Kategorie Herkunft auch die Beschränkung von Rechten verdeutlicht werden. Indem Landnutzer*innen argumentieren, ihre Landrechte aufgrund der ersten Niederlassung ihrer Vorfahren in der Gegend oder der erstmaligen Bestellung des Lands erworben zu haben, fordern indirekt ihre Anerkennung als Indigene ein, um bestehende Rechte an Land gegenüber anderen Akteuren, die nach der Kontrolle über den Zugang zu Land streben, zu untermauern. Ebenso kann der Bezug auf Herkunft - zum Beispiel seitens traditioneller Autoritäten, die Zugang zu Land kontrollieren - dazu dienen, um jemandes Indigenität infrage zu stellen und Zugang zu Land zu beschränken ${ }^{28}$.

Neben Herkunft als Kategorie sozialer Zugehörigkeit sind in beiden Fällen auch Geschlecht und Alter als Analysekategorien relevant für den Zugang $\mathrm{zu}$ Land. Anders als Herkunft führen Landnutzer*innen Geschlecht und Alter jedoch nicht an, um Zugang zu Land zu erlangen oder zu konsolidieren. Vielmehr lassen sich entlang dieser Kategorien Einschränkungen des Zugangs zu Land oder Schwierigkeiten ihn einzufordern oder aufrechtzuerhalten nachvollziehen. Geschlecht ist in beiden Gegenden für die Analyse von Zugang zu Land relevant, da Frauen Zugang zu Land oftmals nur über männliche Familienmitglieder (Vater, Ehemann) erhalten ${ }^{29}$. In der Gegend des BioFuel AfricaProjekts spielen für die geschlechterspezifische Analyse von Zugang zu Land Tätigkeiten eine zentrale Rolle, die ausschließlich Frauen ausüben. $\mathrm{Zu}$ diesen Tätigkeiten zählen die Herstellung von Sheabutter und Dawadawa (einem fermentierten Gewürz aus den Früchten des Johannisbrotbaums) oder das Sammeln von Resten, die nach der maschinellen Ernte durch Landwirt*innen, die das Land zu kommerziellen Zwecken nutzen, auf dem Feld verbleiben ${ }^{30}$.

Alter ist aus folgenden Gründen eine relevante Kategorie für die Analyse des Zugangs zu Land. In der Gegend von ScanFarm berichteten einige Land-

27 Paramount-chief und elders von Tijo, Gruppendiskussion am 8.5.2016; Landnutzerin aus Nsonyameye, Interview am 24.4.2016.

28 Landnutzer*innen und settler-chief aus Kpachaa, Gruppendiskussion am 11.5.2016.

29 Landnutzerin aus Nsonyameye, Interview am 25.4.2016; Research Officer des Regional House of Chiefs der Northern Region, Interview am 8.3.2017; Mitarbeiterin der Civil Society Coalition on Land, Interview am 22.3.2016.

30 Landnutzer*innen und sub-chief von Tuya, Gruppendiskussion am 14.5.2016. 
nutzer*innen, dass sie aufgrund ihres Alters nicht mehr ausreichend darüber informiert seien, wie ihr Land aktuell genutzt würde. Denn sie selbst seien nicht mehr auf den Feldern tätig, sondern diese würden durch ihre Kinder oder durch Pächter*innen bestellt ${ }^{31}$. Zudem hätten sie nicht mehr die Kraft ihre Landrechte aktiv einzufordern, indem sie Unbefugte daran hinderten ihr Land zu nutzen ${ }^{32}$.

Schließlich spielen in beiden Fällen persönliche Beziehungen und Netzwerke eine wichtige Rolle für den Zugang zu Land. Über Beziehungen und Netzwerke kann bestehender Zugang zu Land gefestigt werden und sie erlauben denjenigen Landnutzer*innen Zugang zu Land zu erlangen, die aufgrund ihrer sozialen Zugehörigkeit nicht die Möglichkeit haben, traditionelle Landrechte geltend zu machen. Dies betrifft zum Beispiel junge Menschen - insbesondere Frauen - oder Migrant*innen. In beiden Gegenden haben zahlreiche Landnutzer*innen Zugang zu Land dank familiärer Beziehungen zu Rechteinhaber*innen: junge Menschen erlangen Zugang zu Land zunächst über ihre Eltern, Frauen nach der Heirat oftmals über ihren Mann ${ }^{33}$. Migrant*innen sind auf gute Beziehungen zu indigenen Landnutzer*innen angewiesen, um Zugang zu Land zu erlangen. Gute Beziehungen pflegen sie, indem sie sich in der und für die Gemeinschaft engagieren und sich als vertrauenswürdig erweisen. Dazu gehört, dass sie zu Festlichkeiten in der Gemeinschaft beitragen und ihren Respekt gegenüber traditionellen Autoritäten in Form sporadischer Ernteabgaben zeigen. Auf diese Weise integrieren sie sich in die Gemeinschaft, wodurch sie ihren Zugang zu Land festigen und über mehrere Generationen aufrechterhalten können ${ }^{34}$.

Beziehungen sind in beiden Gegenden wichtig für den Zugang zu Land und insbesondere für die Aufrechterhaltung von Landrechten. In der Gegend von ScanFarm betonen Landnutzer*innen und sub-chiefs die Bedeutung von Respekt und Treue gegenüber den traditionellen Autoritäten, die Zugang zu Land kontrollieren und denen sie ihren Zugang zu Land verdanken ${ }^{35}$. Für vie-

\footnotetext{
31 Landnutzerin aus Nsonyameye, Interview am 25.4.2016.

32 Landnutzerin aus Nsonyameye, Interview am 25.4.2016; Landnutzer aus Dukusen, Interview am 26.4.2016.

33 Mitarbeiterin der Civil Society Coalition on Land, Interview am 22.3.2016.

34 Landnutzer aus Nsonyameye, Interview am 25.4.2016; Landnutzer aus Changolinaaya, Interview am 12.5.2016; Landnutzer und settler-chief aus Parachanayilli, Gruppendiskussion am 13.5.2016.

35 Landnutzer aus Dukusen, Interview am 26.4.2016; sub-chief von Dukusen, Interview am 19.4.2016; sub-chief von Nsonyameye, Interview am 20.4.2016.
} 
le Landnutzer*innen ist das der sub-chief, für viele sub-chiefs der in der Hierarchie übergeordnete paramount chief. Ähnliches gilt auch für Landnutzer*innen in der Gegend des BioFuel Africa-Projekts. Dort ist vor allem für die settler communities die Beziehung zu dem sub-chief, der das Land verwaltet und es ihnen zur Verfügung gestellt hat, wichtig für ihren Zugang zu Land ${ }^{36}$. In der Gegend von ScanFarm sind Beziehungen des Weiteren zentral, um rechtebasierte Zugangsmechanismen wie sharecropping aushandeln zu können. In der Gegend des BioFuel Africa-Projekts trugen zudem persönliche Beziehungen zu Landwirt*innen aus Tamale, die Land für die kommerzielle Landwirtschaft gepachtet hatten, zur Ausweitung des eigenen Zugang zu Land zu bei. Diese Beziehungen ermöglichten es den Landnutzer*innen aus der Gegend Reste, die nach der maschinellen Ernte durch die Landwirt*innen auf den Feldern verblieben, manuell für den Eigenbedarf aufzusammeln ${ }^{37}$.

Netzwerke sind relevant für den Zugang zu Land, da über ihre Mitglieder Wissen und Informationen vermittelt werden, die Einfluss auf Zugang zu Land haben können. Mithilfe von Kenntnissen traditioneller Landrechte gelingt es Landnutzer*innen Argumente zu entwickeln, um Rechte an Land einfordern zu können. Auch für die Anerkennung von Rechten an Land sind Netzwerke relevant, indem ihre Mitglieder beispielweise den Verlauf von Grenzen zwischen den Feldern einzelner Landnutzer*innen oder unterschiedlicher Gemeinschaften bestätigen ${ }^{38}$.

Persönliche und professionelle Netzwerke sowie Netzwerke, die an soziale Positionen geknüpft sind, können ebenfalls Zugang zu Land festigen, indem sie sich vorteilhaft auf andere Zugangsmechanismen auswirken. Dies erwies sich insbesondere im Fall von ScanFarm als relevant. So ermöglichte dort die Mitgliedschaft im ATC oder die gute Beziehung zu Mitgliedern des ATC teilweise privilegierten Zugang zu Informationen bezüglich des Lands sowie finanzielle Zuwendungen, die über die Vergabe von Land generiert werden $^{39}$. Auch professionelle Netzwerke wirkten sich auf den Zugang zu Land

36 Landnutzer aus Changolinaaya, Interview am 12.5.2016; Landnutzer und settler-chief aus Parachanayilli, Gruppendiskussion am 13.5.2016.

37 Landnutzer*innen und sub-chief von Tuya, Gruppendiskussion am 14.5.2016.

38 Landnutzerer aus Nsonyameye und Mitglieder der Agogoman mma kuo, Interview am 16.3.2017; Landnutzer aus Nsonyameye und Vorsitzender der Agogoman mma kuo, Interview am 16.3.2017.

Sub-chief von Dukusen, Interview am 19.4.2016; sub-chief von Nsonyameye, Interview am 20.4.2016; Registerführer des Agogo Traditional Council und Leiter des Agogo Customary Land Secretariat, Interview am 20.4.2016. 
aus. Ein Beispiel dafür sind die Agogoman mma kuo (weltweite Bürger*innen von Agogo), ein Netzwerk, in dem sich Bürger*innen aus Agogo zusammengeschlossen haben, von denen viele lange Zeit im Ausland gelebt haben oder immer noch leben. Ihr Anliegen besteht darin, sich für die Belange der Bewohner*innen von Agogo einzusetzen und die »Entwicklung« Agogos mithilfe internationaler Unterstützung voranzutreiben. Dazu zählt auch die Stärkung von Rechten an Land. Viele Mitglieder dieses Netzwerks haben ein hohes Bildungsniveau, weshalb ein Engagement in diesem Netzwerk den Austausch von Wissen - zum Beispiel in Bezug auf Landrechte - begünstigt und eine gemeinsame Einforderung dieser Rechte - zum Beispiel über das staatliche Rechtssystem - erleichtert ${ }^{40}$. So konnten Netzwerke und soziale Beziehungen Einschränkungen des Zugangs zu Land aufgrund strukturell bedingter Ungleichheitsverhältnisse zum Teil abmildern.

\subsection{Land grabbing und die Veränderung von Kontrolle und Zugang}

Mit der Vergabe des Lands an ScanFarm und BioFuel Africa haben sich die Kontrolle über den Zugang zu Land und die Möglichkeiten Zugang zu Land zu erlangen oder aufrechtzuhalten in beiden Gegenden maßgeblich gewandelt. In erster Linie veränderte sich in diesem Kontext in beiden Fällen die Kontrolle über den Zugang zu Land. Darüber hinaus wurden einige Mechanismen weniger relevant, um Zugang zu Land (aufrecht)erhalten zu können, andere hingegen gewannen im Rahmen der Vergabe des Lands an die Unternehmen an Bedeutung. Wie zu Beginn des Kapitels dargestellt, übten vor der Vergabe des Lands an ScanFarm und BioFuel Africa verschiedene Akteure Kontrolle über den Zugang zu Land aus: der paramount chief, die sub-chiefs sowie Landnutzer*innen mit permanenten Landrechten. Sie konnten in unterschiedlichen Kontexten bestimmen, wer einen Nutzen aus Land ziehen kann. Mit der Vergabe des Lands an die Unternehmen änderte sich dies.

In beiden Fällen entschieden die paramount chiefs über die Vergabe des Lands, ohne sich mit den betroffenen Landnutzer*innen und sub-chiefs zu beraten. Über die Vergabe des Lands an die Unternehmen verloren die sub-chiefs

40 Landnutzerer aus Nsonyameye und Mitglieder der Agogoman mma kuo, Interview am 16.3.2017; Landnutzer aus Nsonyameye und Vorsitzender der Agogoman mma kuo, Interview am 16.3.2017. 
und Landnutzer*innen in beiden Gegenden - wenn auch in unterschiedlichem Ausmaß - die Kontrolle über den Zugang zu ihrem Land. Die paramount chiefs ließen die sub-chiefs und Landnutzer*innen nicht an den Verhandlungen um die Vergabe des Lands teilhaben und gaben ihnen nicht die Möglichkeit, ihre Meinung zu dem Vorhaben zum Ausdruck zu bringen ${ }^{41}$. Damit ignorierten die paramount chiefs indirekt bestehende Rechte und individuelle Vereinbarungen. Im Fall von ScanFarm wusste zu Beginn nur der sub-chief von Dukusen von der Landvergabe, da sein Neffe den Kontakt zu den Investoren hergestellt hatte ${ }^{42}$. Die anderen sub-chiefs sowie die Landnutzer*innen erhielten zunächst keine Informationen. Sie wurden später, als das Unternehmen bereits mit der Nutzung des Lands begonnen hatte, vor vollendete Tatsachen gestellt ${ }^{43}$. Die Landvergabe an BioFuel Africa unterschied sich im Vergleich dazu. Anders als im Fall von ScanFarm setzte sich der Geschäftsführer von BioFuel Africa dafür ein, dass alle Landnutzer*innen aus der Gegend im Rahmen einer öffentlichen Veranstaltung vor Beginn der Landnutzung über das Vorhaben informiert wurden ${ }^{44}$. Diese Maßnahme bedeutete jedoch nicht, dass sub-chiefs und Landnutzer*innen die Möglichkeit bekamen, an den Verhandlungen um die Landvergabe teilzunehmen ${ }^{45}$. Mithilfe von Informant*innen versuchte das Unternehmen allerdings zu erheben, wo sich die Felder der Landnutzer*innen befanden, um diese wenn möglich von der Umnutzung des Lands zu verschonen (Tsikata/Yaro 2011). Welche Landnutzer*innen das

41 Landnutzer*innen aus Nsonyameye, Baama \& Dukusen, Gruppendiskussion am 27.4.2016; Landnutzerin aus Nsonyameye, Interview am 24.4.2016; Landnutzerin aus Nsonyameye, Interview am 24.4.2016; Landnutzer aus Nsonyameye, Interview am 25.4.2016; Landnutzerer aus Nsonyameye und Mitglieder der Agogoman mma kuo, Interview am 16.3.2017; Landnutzerin aus Dukusen, Interview am 16.3.2017; Abteilungsleiterin der Regional Lands Commission der Ashanti Region, Interview am 13.04.2016; Leiter des Department of Agriculture des Asante Akyem North District, Interview am 22.4.2016, Projektleiter des Land and Resource Management Center, Interview am 12.4.2016.

42 Sub-chief von Dukusen, Interview am 19.4.2016.

43 Landnutzerin aus Nsonyameye, Interview am 24.4.2016; Landnutzer aus Nsonyameye, Interview am 25.4.2016; Landnutzerin aus Nsonyameye, Interview am 27.4.2016; Projektleiter des Land and Resource Management Center, Interview am 12.4.2016.

44 Landnutzer*innen und settler-chief aus Kpachaa, Gruppendiskussion am 11.5.2016.

45 Landnutzer*innen und settler-chief aus Kpachaa, Gruppendiskussion am 11.5.2016; Landnutzer*innen und sub-chief von Tuya, Gruppendiskussion am 14.5.2016; Landnutzer aus Changolinaaya, Interview am 12.5.2016. 
Unternehmen oder der paramount chief tatsächlich über das Vorhaben informierten und wessen Felder identifiziert wurden, war mit den Mechanismen verbunden, über die Landnutzer*innen bislang Zugang zu Land erlangten. Migrantische Landnutzer*innen, an die der paramount chief selbst Land vergeben hatte sowie diejenigen aus seiner Gemeinschaft informierte er zeitnah selbst. Indigene sub-chiefs und Landnutzer*innen erhielten die Informationen teilweise über das Unternehmen und teilweise über den paramount chief. Settler communities sowie individuelle Pächter"innen, die ihr Land über einen sub-chief erhalten hatten, waren diejenigen mit dem unsichersten Zugang zu Land. Sie informierte weder der paramount chief noch das Unternehmen.

Im Folgenden analysiere ich entlang der rechtebasierten, relationalen und strukturellen Zugangsmechanismen, inwiefern die Verschiebung der Kontrolle des Zugangs mit einer Veränderung des Zugangs zu Land einhergeht.

\section{Veränderung des Zugangs zu Land über rechtebasierte Mechanismen}

Vor der Vergabe des Lands an ScanFarm und BioFuel Africa waren rechtebasierte Mechanismen in Form traditioneller Landrechte und individueller Vereinbarungen sowie relationale und strukturelle Mechanismen, wie soziale Zugehörigkeit, soziale Beziehungen und Netzwerke die zentralen Mittel, über die Landnutzer*innen Zugang zu Land erlangten. Mit der Verschiebung der Kontrolle des Zugangs weg von den sub-chiefs und Landnutzer*innen hin zu den paramount chiefs verringerten sich die Möglichkeiten der Landnutzer*innen über zuvor relevante Zugangsmechanismen ihren Zugang zu Land aufrechtzuerhalten. In den Verhandlungen um die Vergabe des Lands zwischen den Unternehmen und den paramount chiefs fanden traditionelle Landrechte $\mathrm{zu}$ nächst keine Berücksichtigung, was für die meisten Landnutzer*innen zum Verlust oder zu einer Verschlechterung des Zugangs zu Land führte.

Im Fall von ScanFarm konnten Landnutzer*innen mit permanenten traditionellen Landrechten die Nutzung des Lands nicht mehr selbst kontrollieren. Mit Erlaubnis des paramount chiefs begann das Unternehmen das Land in Abwesenheit der Landnutzer*innen mit Traktoren zu bearbeiten. Auch nach Aufforderung durch die Rechteinhaber*innen, das Land zu verlassen, verfolgte das Unternehmen sein Vorhaben weiter ${ }^{46}$. Arbeiter*innen des Unter- 
nehmens rodeten Bäume in der Nacht, um Widerstand der Landnutzer*innen zu vermeiden ${ }^{47}$. Durch die Arbeiten auf dem Land entfernte das Unternehmen natürliche Grenzmarkierungen, die es Rechteinhaber*innen zuvor ermöglicht hatten, Lage und Größe ihres Landes zu bestimmen ${ }^{48}$. Die einzige Möglichkeit weiterhin einen Nutzen aus ihrem Land ziehen zu können, war die Einforderung von Entschädigungszahlungen. Entschädigungszahlungen konnten Landnutzer*innen, die zuvor über permanente Rechte auf Land verfügten - also Zugang zu Land kontrollierten - einfordern. Sie erhielten diese jedoch nicht automatisch. Das Unternehmen kam nicht direkt auf die Landnutzer*innen zu, um sie für den Verlust ihres Lands zu entschädigen. Um Entschädigungszahlungen in Anspruch nehmen zu können, mussten Landnutzer*innen zunächst ihre zuvor bestehenden traditionellen Rechte beweisen und in diesem Zuge Einschränkungen hinnehmen. Zum Zeitpunkt der Verhandlung über Entschädigungszahlungen hatte das Unternehmen Grenzmarkierungen bereits entfernt oder zerstört. Aufgrund dieser fehlenden Grenzmarkierungen war es für viele Landnutzer*innen schwierig, die Lage ihres Landes und damit einhergehend dessen Größe zu bestimmen. In der Berechnung der Entschädigungszahlungen berücksichtigte das Unternehmen daher oftmals nur Flächen die kleiner waren, als diejenigen, an denen Landnutzer*innen zuvor traditionelle Landrechte hatten ${ }^{49}$. Das Unternehmen zahlte Landnutzer*innen einen einmaligen Betrag von 30 GHS pro acre sowie eine jährliche Entschädigungssumme von 10 GHS pro acre. Gemessen an dem zuvor über das Land generierte Einkommen waren die Entschädigungszahlungen darüber hinaus für viele Landnutzer*innen nachteilig $^{50}$. Diejenigen, die ihr Land vor der Vergabe an das Unternehmen ver-

47 Landnutzerin aus Nsonyameye, Interview am 27.4.2016; Landnutzerin aus Dukusen, Interview am 16.3.2017; Landnutzer aus Nsonyameye und Vorsitzender der Agogoman mma kuo, Interview am 16.3.2017.

48 Landnutzer"innen aus Nsonyameye, Baama \& Dukusen, Gruppendiskussion am 27.4.2016; Landnutzerin aus Nsonyameye, Interview am 24.4.2016; Landnutzerin aus Nsonyameye, Interview am 26.4.2016; Landnutzerin aus Nsonyameye, Interview am 27.4.2016; Landnutzer aus Nsonyameye und Vorsitzender der Agogoman mma kuo, Interview am 16.3.2017.

49 Landnutzerin aus Nsonyameye, Interview am 26.4.2016; Landnutzer aus Nsonyameye, Interview am 28.4.2016; Landnutzerer aus Nsonyameye und Mitglieder der Agogoman mma kuo, Interview am 16.3.2017; Landnutzer aus Nsonyameye und Vorsitzender der Agogoman mma kuo, Interview am 16.3.2017.

50 Landnutzerin aus Nsonyameye, Interview am 24.4.2016; Landnutzerin aus Nsonyameye, Interview am 24.4.2016; Landnutzerin aus Nsonyameye, Interview am 25.4.2016; 
pachtet hatten, erhielten 200 GHS Pacht pro acre und Jahr ${ }^{51}$. Im Rahmen von sharecropping-Vereinbarungen bekamen sie einen Teil der Ernte, der vom Wert vergleichbar mit Pachteinnahmen war, wie einen Sack Mais pro acre ${ }^{52}$. Die Entschädigungszahlungen durch das Unternehmen waren somit 20-mal geringer. Trotz bestehender Rechte, die das Unternehmen durch die Leistung von Entschädigungszahlungen anerkannte, verschlechterte sich der Zugang zu Land im Kontext der Landvergabe. Der finanzielle Nutzen, den Landnutzer*innen über die Entschädigungszahlungen aus dem Land zogen, war deutlich geringer ist als derjenige, den sie zuvor durch das Land hatten.

Für Landnutzer*innen, deren Rechte an die Nutzung des Lands gebunden waren - zum Beispiel im Rahmen individueller Vereinbarungen - hatte die Veränderung der Zugangskontrolle zur Folge, dass sie ihren Zugang $\mathrm{zu}$ Land vollständig verloren. Im Gegensatz zu Landnutzer*innen mit permanenten Rechten waren ihre Ansprüche auf Entschädigungszahlungen sehr gering: das Unternehmen entschädigte diese Landnutzer*innen nur für den Verlust ihrer Ernte. Wenn sie das Land in dem Zeitraum, in dem es vergeben wurde, jedoch nicht bestellt hatten - weil zum Beispiel keine Anbausaison war - oder sie nicht beweisen konnten, dass sie ihre Ernte durch die Arbeiten des Unternehmens verloren hatten, erhielten sie keine Entschädigung ${ }^{53}$.

Vorteilhaft waren die Entschädigungszahlungen nur für ältere Landnutzer*innen, die ihr Land vor der Vergabe an ScanFarm nicht mehr selbst genutzt oder verpachtet, sondern es brach liegen lassen hatten. Für sie bedeuteten die Entschädigungszahlungen ein, wenn auch geringes, regelmäßiges Einkommen und damit eine Verbesserung des Zugangs zu Land ${ }^{54}$. Land, das lange Zeit brach liegt, ist jedoch selten ungenutzt. Mitglieder der Gemeinschaft nutzen es für vielfältige Zwecke.

Eine weitere Veränderung rechtebasierter Zugangsmechanismen, die mit der Verschiebung der Zugangskontrolle einherging, ist die neue Bedeutung, die formalisierte traditionelle Landrechte für Zugang zu Land bekommen.

Landnutzer aus Nsonyameye, Interview am 25.4.2016; Landnutzerin aus Nsonyameye, Interview am 26.4.2016; Landnutzerin aus Nsonyameye, Interview am 27.4.2016; Landnutzerin aus Nsonyameye, Interview am 27.4.2016.

51 Landnutzer aus Nsonyameye, Interview am 25.4.2016; Landnutzerin aus Nsonyameye, Interview am 27.4.2016; Landnutzerin aus Nsonyameye, Interview am 27.4.2016; Landnutzer*innen aus Nsonyameye, Baama \& Dukusen, Cruppendiskussion am 27.4.2016.

52 Landnutzer aus Nsonyameye, Interview am 25.4.2016.

53 Landnutzer aus Nsonyameye, Interview am 25.4.2016.

54 Sub-chief von Nsonyameye, Interview am 20.4.2016. 
Vor der Vergabe des Lands an ScanFarm hatte die Formalisierung traditioneller Landrechte keinen direkten Nutzen für den Zugang zu Land. Um die Kontrolle über den Zugang zu Land nach der Vergabe des Lands wieder zurückgewinnen $\mathrm{zu}$ können, erwiesen sich formalisierte traditionelle Landrechte jedoch als unabdingbar. Im Vergleich $\mathrm{zu}$ nicht-formalisierten traditionellen Rechten sind sie leichter zu beweisen. Dennoch verloren auch Landnutzer*innen mit formalisierten Rechten im Rahmen der Landvergabe die Kontrolle über den Zugang zu ihrem Land. Da das Land - wenn auch ohne das Wissen der Rechteinhaber*innen - Teil des Pachtvertrags war, erhob das Unternehmen Anspruch darauf ${ }^{55}$. Zwar bestätigte der paramount chief gegenüber den Landnutzer*innen ihre formalisierten Rechte, setzte sie jedoch nicht gegenüber dem Unternehmen durch. Somit bewirkte die Formalisierung keine automatische Anerkennung der Rechte durch das Unternehmen. Die einzige Möglichkeit die Rechteinhaber* innen blieb, um ihre Landrechte einzufordern, war der staatliche Rechtsweg, also eine Klage gegen die Nutzung des Lands durch das Unternehmen ${ }^{56}$. Landnutzer*innen ohne formalisierte Landrechte, also ohne Landrechte die durch traditionelle Autoritäten schriftlich bestätigt waren, hatten diese Möglichkeit nicht. Das Gerichtsverfahren am Kumasi High Court begann im Januar 2014 und war zur Zeit des letzten Interviews im März 2017 noch nicht entschieden ${ }^{57}$. Formalisierte Landrechte ermöglichten Landnutzer*innen somit zwar für ihre Rechte zu kämpfen, sicherten jedoch keinen direkten Zugang zu Land.

Durch die Einforderung von Landrechten über das staatliche Rechtssystem gewannen staatliche Akteure an Einfluss in der Durchsetzung traditioneller Rechte. Zuvor hatten diese keinerlei Einfluss auf traditionelle Landrechte. Im Kontext der Landvergabe ist die Einbeziehung staatlicher Akteure (Richter*innen, Staatsanwält"innen) jedoch unabdingbar, um zuvor bestehenden Zugang zu Land über traditionelle Rechte durchsetzen zu können. Um das Unternehmen erfolgreich daran hindern zu können, Land - das nach

55 Landnutzerer aus Nsonyameye und Mitglieder der Agogoman mma kuo, Interview am 16.3.2017; Landnutzer aus Nsonyameye und Vorsitzender der Agogoman mma kuo, Interview am 16.3.2017.

56 Landnutzerer aus Nsonyameye und Mitglieder der Agogoman mma kuo, Interview am 16.3.2017; Landnutzer aus Nsonyameye und Vorsitzender der Agogoman mma kuo, Interview am 16.3.2017.

57 Landnutzer aus Nsonyameye und Vorsitzender der Agogoman mma kuo, Interview am 16.3.2017. 
Meinung des Unternehmens offiziell Teil des Pachtvertrags war - mit schweren Maschinen zu bearbeiten, benötigen Landnutzer"innen einen Beweis in Form eines staatlich bestätigten Dokuments, das ihre Rechte an diesem Land belegte

Die Landvergabe an BioFuel Africa unterschied sich von derjenigen an ScanFarm. Gleich ist jedoch, dass sich auch in diesem Fall die Kontrolle des $\mathrm{Zu}$ gangs zu Land weg von sub-chiefs und Landnutzer*innen hin zum paramount chief verschob. Anders als im Fall von ScanFarm setzte sich das Unternehmen nach Rücksprache mit dem paramount chief und staatlichen Akteuren wie Mitarbeiter* innen der Environmental Protection Agency (EPA) und der Regional Lands Commission dafür ein, dass Landnutzer*innen mit traditionellen Rechten an dem Land über das Vorhaben informiert und ihre Landrechte berücksichtigt werden (Bruce 2008; SGS Environment 2009; Tsikata/Yaro 2011). Im Rahmen einer Informationsveranstaltung in Kumasi, zu der chiefs und Landnutzer* innen eingeladen waren, informierte das Unternehmen über das geplante Vorhaben und gab den Anwesenden die Möglichkeit, Fragen diesbezüglich zu stellen. Darüber hinaus strebte es an, die genutzten Felder zu identifizieren. Wenn möglich wollte es diese von ihrem Vorhaben verschonen oder ansonsten eine Alternative zur Verfügung zu stellen, indem es bislang unbestelltes Land außerhalb der gepachteten Fläche für die Landnutzer*innen rodete und pflügte (Bruce 2008; Tsikata/Yaro 2011). Die Unternehmenspolitik ermöglichte es indigenen Landnutzer*innen mit permanenten Landrechten im Rahmen der Vergabe des Lands an BioFuel Africa ihren eigenen Zugang zu Land aufrechtzuerhalten. Das an das Unternehmen vergebene Land ist relativ dünn besiedelt und die meisten Dörfer sind sehr klein. Das ermöglichte dem Unternehmen die Felder für den Jatropha-Anbau so anzulegen, dass es die Dörfer sowie die um die Dörfer liegenden Felder der Landnutzer*innen aussparte und dennoch eine zusammenhängende Fläche bewirtschaften konnte (SGS Environment 2009).

Eine maßgebliche Beschränkung des Zugangs zu Land zog die Verschiebung der Zugangskontrolle jedoch für Nutzer*innen nach sich, die nicht über permanente, individuelle Rechte an Land verfügen, sondern Zugang $\mathrm{zu}$ gemeinschaftlich genutztem Land über kollektive Rechte hatten. Dieses Land zeichnete sich dadurch aus, dass eine Vielzahl an wirtschaftlich nutzbaren Bäumen darauf wuchs. In Vorbereitung der gepachteten Fläche für den Jatropha-Anbau rodete das Unternehmen Teile dieses gemeinschaftlich genutzten Lands um die Dörfer Kpachaa, Tuya, Jashei und Parachanayil- 
$\mathrm{li}^{58}$. Dies schränkte Zugang zu Land insbesondere für Frauen ein, da diese ihr Haupteinkommen durch die Verarbeitung von Baumfrüchten und den Verkauf der Endprodukte erwirtschafteten. Durch die Rodung der Bäume verloren sie diese Einkommensquelle. Während Landnutzer"innen mit permanenten Landrechten im Verlustfall ein alternatives Stück Land erhielten, räumte das Unternehmen Nutzer*innen mit kollektiven Rechten keinen Anspruch auf eine derartige Entschädigung ein. Gleiches galt für Migrant*innen, settler communities sowie Pächter*innen, die das Land für die kommerzielle Landwirtschaft nutzten, jedoch nicht vor Ort lebten. Sie alle hatten die Möglichkeit, sich eigenständig um ein neues Stück Land $\mathrm{zu}$ bemühen, konnten nach traditionellem Recht jedoch keinen Anspruch darauf erheben ${ }^{59}$. Da ihnen die Landnutzungsrechte jederzeit entzogen werden konnten, erhielten sie - solange sie ihre Ernte durch die Vergabe des Lands nicht verloren - auch keine Entschädigungszahlungen seitens des Unternehmens.

\section{Veränderung des Zugangs zu Land über relationale und strukturelle Mechanismen}

Vor der Vergabe des Lands an ScanFarm und BioFuel Africa ermöglichten oder festigten unterschiedliche Formen der Aushandlung von Landnutzungsrechten innerhalb sozialer Beziehungen Zugang zu Land. Landnutzer*innen erlangten Zugang zu Land durch Rückbezug auf Kategorien sozialer Zugehörigkeit, persönliche Beziehungen zu Rechteinhaber*innen oder das Engagement in professionellen Netzwerken. Im Kontext der Vergabe des Lands an ScanFarm und BioFuel Africa spielen relationale Mechanismen weiterhin eine Rolle für den Zugang zu Land. Durch die Verschiebung der Kontrolle des Zugangs verloren viele Mechanismen an Bedeutung. Nach der Vergabe des Lands an die Unternehmen waren die für Zugang zu Land bedeutsamen relationalen Zugangsmechanismen somit weniger divers als

58 Landnutzer*innen und settler-chief aus Kpachaa, Gruppendiskussion am 11.5.2016; Landnutzer und settler-chief aus Parachanayilli, Gruppendiskussion am 13.5.2016; Landnutzer*innen und sub-chief von Tuya, Gruppendiskussion am 14.5.2016; Landnutzer*innen und sub-chief von Jashei, Gruppendiskussion am 15.5.2016.

59 Landnutzer*innen und settler-chief aus Kpachaa, Gruppendiskussion am 11.5.2016; Landnutzer und settler-chief aus Parachanayilli, Gruppendiskussion am 13.5.2016; Research Officer des Regional House of Chiefs der Northern Region, Interview am 8.3.2017; I-StA-12. 
vorher. Sie beschränkten sich im Kern auf Beziehungen zu traditionellen Autoritäten, Beziehungen zu den Unternehmen sowie die erfolgreiche Anerkennung als indigene Landnutzer*innen durch Akteure, die Zugang zu Land kontrollieren, wie die paramount chiefs oder Unternehmen.

Die Einschränkung der rechtebasierten Zugangsmechanismen, die mit der Verschiebung der Kontrolle von Zugang zu Land einherging, wirkte sich ebenfalls auf relationale Zugangsmechanismen aus. Da sub-chiefs und Landnutzer*innen mit permanenten Landrechten die Kontrolle über den Zugang zu Land verloren, konnten persönliche Beziehungen $\mathrm{zu}$ ihnen und daraus hervorgehende mündliche Vereinbarungen keinen Zugang zu Land mehr gewährleisten. Nur Vereinbarungen, die mit dem paramount chief getroffen wurden, blieben weiterhin von Relevanz. Im Gegenzug dazu gewann soziale Zugehörigkeit an Bedeutung. Migrantische Landnutzer*innen sowie Pächter*innen aus anderen Gegenden waren die Verlierer*innen der Landvergabe. Sie haben keinen Anspruch darauf, für den Verlust von Zugang zu Land finanziell oder über die Bereitstellung von Alternativflächen entschädigt zu werden. Aber auch andere relationale und strukturelle Mechanismen gewannen an Relevanz für den Zugang zu Land. Wissen und Bildung gewannen beispielsweise im Kontext der Landvergabe an Bedeutung. Insbesondere der Fall der Landvergabe an ScanFarm lässt darauf schließen, dass Informationen zur Vergabe des Lands, Kenntnisse der eigenen Rechte und Bildung allgemein grundlegend waren, um Zugang zu Land aufrechtzuerhalten oder zurückzufordern. Bildung spielte eine zentrale Rolle für die Einforderung von Landrechten per Gerichtsverfahren, um Briefe an das Unternehmen, den paramount chief und Behörden schreiben und auf Landrechte aufmerksam machen zu können, um schriftliche Informationen zum Projekt von Behörden- oder Unternehmensseite lesen zu können und den Inhalt des Memorandum of Understanding bezüglich der Entschädigungszahlungen an die Landnutzer*innen durch das Unternehmen verstehen $\mathrm{zu}$ können ${ }^{60}$. Einige Interviews verdeutlichten, dass insbesondere ältere Personen mit einem schwachen Bildungshintergrund den Inhalt und die Reichweite des

60 Landnutzerer aus Nsonyameye und Mitglieder der Agogoman mma kuo, Interview am 16.3.2017; Landnutzer aus Nsonyameye und Vorsitzender der Agogoman mma kuo, Interview am 16.3.2017; Brief des Registerführers des ATC an den Geschäftsführer von ScanFarm, 4.1.2010; Brief der Landnutzerinnen von Baama an den Vorsitzenden der Asante Akyem North Distric Assembly, 1.2.2011; Brief der Landnutzerinnen von Baama an den Vorsitzenden der Asante Akyem North Distric Assembly, 16.2.2011. 
Memorandum of Understanding nicht gänzlich verstanden ${ }^{61}$. Ihnen war nicht klar, dass das Unternehmen eine einmalige Entschädigungszahlung pro acre Land leistet, die höher ist als die jährliche Zahlung. Sie gingen davon aus, dass sie diese einmalige Zahlung von 30 GHS pro acre von nun an jährlich erhalten würden, wobei die jährliche Entschädigungszahlung nur ein Drittel davon betrug. Darüber hinaus konnten sie nicht nachvollziehen, wie sich die Entschädigungszahlungen zusammensetzten und wie das Unternehmen die Größe ihres Lands berechnet hatte ${ }^{62}$. Im Kontext der Landvergabe sind ungleiche Möglichkeiten Zugang zu Land aufrechtzuhalten oder zurückzufordern somit stärker als vorher an soziale Zugehörigkeit und damit einhergehende soziale Differenzierung und strukturelle Ungleichheitsverhältnisse geknüpft.

Aufgrund der Aneignung der Kontrolle über Land durch die paramount chiefs, wurde im Kontext der Landvergabe die Beziehung zu ihnen zum zentralen Zugangsmechanismus. Sie wirkt sich auf den Erhalt und den Umfang von Informationen in Bezug auf die Vergabe des Lands aus und ermöglicht eine finanzielle Beteiligung an den Einnahmen aus der Landvergabe. Darüber hinaus führte eine enge Beziehung zum paramount chief zu privilegiertem $\mathrm{Zu}$ gang zu Arbeit für das Unternehmen oder Serviceleistungen, wie das maschinelle Roden oder Pflügen neuer Flächen durch das Unternehmen ${ }^{63}$ (Bruce 2009).

Im Fall von ScanFarm ermöglichte eine gute Beziehung zum paramount chief zeitnahe Informationen zu dem agrarindustriellen Vorhaben des Unternehmens ${ }^{64}$. So erfuhr zunächst der sub-chief von Dukusen von dem Vorhaben, da sein Neffe, die Beziehung zwischen dem paramount chief und den Investoren hergestellt hatte ${ }^{65}$. Darüber hinaus war die Beziehung zum paramount chief zentral dafür, wen er mit der Aufgabe betraute, dem Unterneh-

61 Landnutzerin aus Nsonyameye, Interview am 24.4.2016; Landnutzerin aus Nsonyameye, Interview am 26.4.2016; Landnutzerin aus Nsonyameye, Interview am 27.4.2016.

62 Landnutzerin aus Nsonyameye, Interview am 26.4.2016; Landnutzerin aus Nsonyameye, Interview am 27.4.2016.

63 Sub-chief von Baama, Interview am 20.04.2016, Registerführer des Agogo Traditional Council und Leiter des Agogo Customary Land Secretariat, Interview am 20.4.2016.

64 Sub-chief von Dukusen, Interview am 19.4.2016; sub-chief von Nsonyameye, Interview am 20.4.2016; sub-chief von Baama, Interview am 20.04.2016.

65 Landnutzer aus Dukusen, Interview am 26.4.2016; sub-chief von Dukusen, Interview am 19.4.2016; sub-chief von Baama, Interview am 20.04.2016; Registerführer des Agogo Traditional Council und Leiter des Agogo Customary Land Secretariat, Interview am 20.4.2016. 
men das zur Verfügung stehende Land zu zeigen. Aufgrund ihres Vertrauensverhältnisses erhielt der ehemalige sub-chief von Dukusen diese Aufgabe und nicht, wie eigentlich üblich, der amtierenden sub-chief ${ }^{66}$. Des Weiteren wirkte sich die Beziehung zu Mitgliedern des ATC positiv auf den Zugang zu Arbeit für ScanFarm aus. Während ScanFarm nur wenige Landnutzer*innen aus den Dörfern als Hilfsarbeiter*innen auf saisonaler Basis einstellte, erhielt der Sohn des Registrators (registrar) des ATC eine permanente Einstellung als Fahrer für das Unternehmen, die Tochter des paramount chiefs arbeitete im Management des Unternehmens und der ehemalige sub-chief von Dukusen erhielt eine Festanstellung als Arbeiter ${ }^{67}$. Andere Zugangsmechanismen wie Beziehungen zu individuellen Rechteinhaber*innen und daraus hervorgehende Vereinbarungen zur Nutzung von Land konnten nach der Vergabe des Lands an das Unternehmen keinen Zugang zu Land mehr gewährleisten.

Auch im Fall von BioFuel Africa spielte im Kontext der Landvergabe die Beziehung zum Tijo Naa, dem dortigen paramount chief eine entscheidende Rolle für den Zugang zu Land der Landnutzer*innen. Sie hatte Einfluss darauf, ob und zu welchem Zeitpunkt der Tijo Naa die landnutzenden Gemeinschaften über die Umnutzung des Lands informierte oder ob er das Unternehmen auf die Existenz der Dörfer hinwies, damit es diese informieren, ihre Felder entsprechend lokalisieren und bei den Arbeiten aussparen konnte. Der settler chief von Kpachaa, der sein Land über den Tijo Naa erhalten hatte, wurde zeitnah durch den Tijo Naa informiert, während die sub-chiefs von Jashei und Jimle erst einige Zeit später durch die Investoren selbst von dem Projekt erfuhren, obwohl sie Indigene sind und damit permanente Landrechte haben ${ }^{68}$. Entsprechend konnten auch sie die Informationen erst später an die settler communities auf ihrem Land weitergeben. Der Informationsfluss wurde damit willkürlich durch den paramount chief beeinflusst und Beziehungen, die vorher für individuelle Vereinbarungen wichtig waren - zum Beispiel zu den sub-

66 Landnutzer*innen aus Nsonyameye, Baama \& Dukusen, Gruppendiskussion am 27.4.2016; Landnutzer aus Dukusen, Interview am 26.4.2016; Landnutzerin aus Nsonyameye, Interview am 26.4.2016; Landnutzerin aus Nsonyameye, Interview am 27.4.2016; Landnutzerin aus Nsonyameye, Interview am 27.4.2016.

67 Registerführer des Agogo Traditional Council und Leiter des Agogo Customary Land Secretariat, Interview am 20.4.2016; sub-chief von Baama, Interview am 20.04.2016.

68 Landnutzer*innen aus jimle und Cumani, Gruppendiskussion am 10.5.2016; Landnutzer*innen und settler-chief aus Kpachaa, Gruppendiskussion am 11.5.2016; Landnutzer"innen und sub-chief von Jashei, Gruppendiskussion am 15.5.2016. 
chiefs - konnten Zugang zu Land nicht mehr gewährleisten. Darüber hinaus sei der Großteil der Arbeitsplätze, die BioFuel Africa schuf, an Familienmitglieder, Freund*innen und Bekannte des Tijo Naas aus Tamale vergeben worden, wo der Tijo Naa seinen Hauptwohnsitz hat (Kwoyiga 2012: 46). Klientelismus gewinnt damit in beiden Fällen maßgeblich an Bedeutung.

Aufgrund des Verlusts oder der Einschränkung von Zugang setzten Landnutzer*innen in beiden Gegenden große Hoffnungen in eine Anstellung bei den Unternehmen. Lohnarbeit ermöglichte in beiden Gegenden jedoch nur einer geringen Zahl von Landnutzer*innen Zugang zu Land, der darüber hinaus nur temporär oder sehr unsicher war, da das Unternehmen Arbeitsverhältnisse jederzeit aufkündigen konnte. ScanFarm stellte insgesamt 80 Personen ein, von denen 30 permanent beschäftigte Fachkräfte waren, die nicht aus den Dörfern in der Projektgegend kamen. 50 Angestellte waren temporär beschäftigte Hilfs- und Gelegenheitsarbeiter*innen (Erntehelfer*innen, Pestizidsprüher*innen, Pflanzer*innen) aus den Dörfern, die das Unternehmen ein bis drei Monate im Jahr beschäftigte. Sie erhielten pro Tag einen Lohn von 15 bis 20 GHS, was damals ca. 9 bis 12 Euro entsprach (Boamah/Overå 2016: 122). BioFuel Africa beschäftigte während des kurzen Höhepunkts des Projekts im Jahr 2009400 Angestellte (Persönliche Kommunikation CEO BioFuel Africa). Aus den Dörfern in der Projektgegend kamen jedoch nur ca. 70-80 Personen und 50 davon alleine aus Jimle ${ }^{69}$. Der Großteil der Angestellten aus den Dörfern waren Männer, von denen die meisten saisonal als Pestizidsprüher oder Feldarbeiter arbeiteten. Einige bekamen Posten als Aufseher und Wachmänner. Die wenigen Frauen, die das Unternehmen beschäftigte, arbeiteten als Ernte- und Pflanzhelferinnen. Je nach Position erhielten sie einen Lohn von 65 bis 200 GHS pro Monat. Das entsprach zu diesem Zeitpunkt ca. 40 bis 120 Euro. Feldarbeiter*innen bekamen mit 65 GHS den geringsten Lohn, während die Aufseher mit 200 GHS pro Monat den höchsten Lohn erhiel$\operatorname{ten}^{70}$.

69 Landnutzer*innen aus Jimle und Gumani, Gruppendiskussion am 10.5.2016.

70 Landnutzer*innen aus Jimle und Cumani, Gruppendiskussion am 10.5.2016; Landnutzer und settler-chief aus Parachanayilli, Gruppendiskussion am 13.5.2016; Landnutzer*innen und sub-chief von Jashei, Gruppendiskussion am 15.5.2016. 


\subsection{Zwischenfazit}

Vor der Vergabe des Lands an die Unternehmen ScanFarm und BioFuel Africa teilte sich die Kontrolle des Zugangs zu Land in beiden Gegenden zwischen unterschiedlichen Akteuren auf. In beiden Fällen kontrollierten der paramount chief, die sub-chiefs und Landnutzer*innen mit permanenten Rechten Zugang $\mathrm{zu}$ Land. Sie konnten darüber entscheiden, wer Land wie nutzen konnte. Die Möglichkeit dieser Akteure Zugang zu Land zu kontrollieren, leitete sich aus ihrer Herkunft ab; aus der Anerkennung als Indigene, deren Vorfahren bereits aus der Gegend stammten und Land urbar gemacht oder die das Land erstmalig bestellt hatten.

Landnutzer*innen, die Zugang zu Land nicht eigenständig kontrollierten, erlangten Zugang über rechtebasierte, relationale und strukturelle Zugangsmechanismen, die in einem wechselseitigen Verhältnis zueinander standen. Rechtebasierte Zugangsmechanismen wie traditionelle Landrechte oder individuelle Vereinbarungen waren mit relationalen und strukturellen Mechanismen wie sozialer Zugehörigkeit, sozialen Beziehungen und Netzwerken verknüpft. Landnutzer*innen, die Zugang zu Land über traditionelle Rechte erlangten, konnten diese rechtebasierten Zugangsmechanismen über relationale und strukturelle Zugangsmechanismen stärken. Landnutzer*innen, die aufgrund ihrer sozialen Zugehörigkeit keine traditionellen Landrechtsansprüche geltend machen konnten, hatten die Möglichkeit, Zugang zu Land mittels relationaler und struktureller Zugangsmechanismen zu erlangen.

Mit der Vergabe des Lands an die Unternehmen veränderte sich in beiden Fällen zunächst die Kontrolle über den Zugang zu Land. Die Verhandlungen über die Vergabe des Lands führten die Unternehmen mit den paramount chiefs und ihren elders. Die sub-chiefs und Landnutzer*innen mit permanenten Landrechten erhielten nicht die Möglichkeit an den Verhandlungen teilzunehmen und Entscheidungen mitzubestimmen. Dadurch verloren sie die Kontrolle über den Zugang zu Land an die paramount chiefs. Aufgrund der Verschiebung der Kontrolle weg von den sub-chiefs und Landnutzer*innen hin zu den paramount chiefs veränderten sich auch die Möglichkeiten Zugang zu Land zu erlangen. Landnutzer*innen, die Zugang zu Land bislang über Beziehungen $\mathrm{zu}$ anderen Akteuren als den paramount chief erlangt hatten, konnten diesen im Kontext der Landvergabe nicht aufrechterhalten. Traditionelle Landrechte und individuelle Vereinbarungen verloren maßgeblich an Bedeutung und ermöglichten es Landnutzer*innen kaum noch, einen Nutzen aus dem Land zu ziehen. Stattdessen gewannen - insbesondere im Fall von ScanFarm - staat- 
lich registrierte Rechte an Relevanz. Nach der Vergabe des Lands erlangten Akteure zudem in beiden Fällen zunehmend Zugang zu Land über klientelistische Beziehungen. 
